\title{
Influence of Chelated Ca, Micronutrient and Method of Application on Vegetative Growth of Pomegranate (Punica granatum L.) cv. Bhagwa
}

\author{
Vandana $^{1 *}$, G.M. Waghmare ${ }^{1}$ and Shivendu Pratap Singh Solanki ${ }^{2}$ \\ ${ }^{1}$ Department of Horticulture, Vasantrao Naik Marathwada Agricultural University, \\ Parbhani, M.S., India \\ ${ }^{2}$ Department of Fruit Science, Dr Y S Parmar University of Horticulture and Forestry, \\ Nauni, Solan, H.P., India \\ *Corresponding author
}

\begin{tabular}{|c|c|}
\hline \multicolumn{2}{|r|}{ A B S T R A C T } \\
\hline & \multirow{7}{*}{$\begin{array}{l}\text { Investigation was undertaken to study the effect of pre-harvest sprays of some } \\
\text { mineral nutrients on the vegetative growth of pomegranate. The experiment was } \\
\text { laid out in factorial randomized block design with two factors i.e. different } \\
\text { concentration of chelated Ca, micronutrient (Grade) and method of application. } \\
\text { These factors consist of six and three levels respectively, eighteen treatment } \\
\text { combination and two replications. The experiment was conducted on two and half } \\
\text { years old pomegranate plants of Bhagwa cultivar in the experimental fruit orchard } \\
\text { of Department of Horticultural, V.N.M.K.V., Parbhani, Dist- Parbhani during } \\
\text { ambe bahar in 2015-16. The micronutrient mixtures i.e. chelated Ca ( } 9 \% \text { ) and } \\
\text { Chelated micronutrient were applied peak vegetative growth, second spray 50\% } \\
\text { flowering stage and third spray fruit development stage by foliar, soil and foliar + } \\
\text { soil application method respectively. }\end{array}$} \\
\hline Keywords & \\
\hline $\begin{array}{l}\text { Chelated Ca, } \\
\text { Micronutrients, } \\
\text { Ambe bahar }\end{array}$ & \\
\hline Article Info & \\
\hline $\begin{array}{l}\text { Accepted: } \\
\text { 04 September } 2017\end{array}$ & \\
\hline $\begin{array}{l}\text { Available Online: } \\
10 \text { November } 2017\end{array}$ & \\
\hline & \\
\hline
\end{tabular}

\section{Introduction}

The pomegranate (Punica granatum Linn) is one of the oldest fruits that have not changed much throughout the history this fruit was one of the first five crops along with Figs, Dates, Olives and Grapes to be cultivated. Pomegranate is a tropical and subtropical fruit. Its domestication started 3000 - 4000 BC in the North of Iran and Turkey (Lye, 2008) from where it spread to other regions e.g. Mediterranean countries, India and China, possibly through ancient trade routes. Pomegranates have been variously placed in the Punicaceae family, depending on the taxonomist and whether they are considering morphological or molecular data. Its name in Latin means "apple with many seeds". The fruit, which is about the size of an apple, has a thin rind lined with a layer of white membrane which divides the fruit into several cells. Each cell contains numerous seeds encased in a juicy pulp.

The fruit size can vary from $6-12 \mathrm{~cm}$ in diameter and has a tough, leathery skin. The outer rind color is pink to deep red when fruit is mature on the most popular varieties for 
eating. Each aril contains a seed surrounded by edible juicy pulp.

Many investigations studied the effect of application of macro and micronutrients on growth, yield and fruit. However boron, zinc and calcium were highly Fruit cracking seems to be a problem that lessens application of some micronutrients such as $\mathrm{Fe}, \mathrm{B}, \mathrm{Mn}$ and $\mathrm{Cu}$. the $\mathrm{Zn}$ increased fruit yield, whereas B reduced the percentage of cracked fruits. Foliar application is method for reducing the use of chemical fertilizers (Malakouti and Tabatabaei, 1999).

\section{Materials and Methods}

The experiment was carried out during 201516 in ambe bahar the field consisted of 144 plants of cv. Bhagwa, planted in june 2013 having age of two and half years at the spacing of $4 \times 3 \mathrm{~m}$. Four uniform and healthy plants were selected from the field for each replication.The said experiments were replicated twice. Five fruits from a single tree were randomly selected to study the physical and chemical composition of cv. Bhagwa.

The field experiment was conducted at Department of Horticulture, V.N.M.K.V. Parbhani. The area is dominated by black soils which are formed from basaltic material originating through volcanic eruptions. The soil is characterized by black colour dominated by montmorillonite clay with high coefficient of expansion when wet and shrinkage in summer leading to deep cracking, the soils are alkaline in reaction with high base saturation. According to $7^{\text {th }}$ approximation the soils are classified as Typic chromoster Malewar (1977) and included in Parbhani series. The experiment was laid out in Factorial Randomized Block Design (FRBD) and it consisted of spraying of different concentration of different type of chelated $\mathrm{Ca}$ and micronutrient
The Micronutrient was applied by foliar spray and root zone of plants with the help of knapsack sprayer and application through soil was done in fertilizer ring care was also taken to avoid spray of one solution over other treatments. The spray was washed with the clean water after the application of every solution of chelated $\mathrm{Ca}$ and Micronutrient and with the standardize dose of Calcium Chelate to prepare $0.05 \%$ stock solution of Calcium Chelate, take weight $0.5 \mathrm{gm}$ calcium chelate in a weighing balance, put in volumetric flask and dissolved in water to make the volume up to 1 litre.

Similarly, $0.1 \%, \quad 0.15 \% \quad$ calcium chelate solution prepared and this solution is used for spraying and Chelated Micronutrient to prepare $0.20 \%$ stock solution of Chelated Micronutrient, take weight $2 \mathrm{gm}$ of chelated micronutrient in a weighing balance, put in volumetric flask and dissolved in water to make the volume up to 1 litre. Similarly, $0.25 \%$ and $0.30 \%$ chelated Micronutrient solution Prepared and this solution is used for spraying.

The growth of plants observed under different treatment was recorded. The height of the plant was measured with the help of measuring tape from ground level to top of the main branch or leader before and after spraying and expressed in centimetres, The number of branches were calculated before and after spraying of chelated $\mathrm{Ca}$ and micronutrient on every selected plant and Spread of plant in N-S and E-W direction and Girth of stem is measured by measuring tape above the ground and The number of leaves were calculated before and after spraying of chelated $\mathrm{Ca}$ and micronutrient. The data obtained in respect of various observations were subjected to the statistical analysis (Factorial Randomized Block Design) as per the procedure given by Panse and Sukhatme (1989). 


\section{Results and Discussion}

\section{Growth parameters}

\section{Height of plant}

Data on percent increment in height of plant of pomegranate were influenced by due to treatments of chelated $\mathrm{Ca}$, micronutrients and methods of application as presented in table 1 .

The data presented in table 1 indicated that, different concentration of chelated $\mathrm{Ca}$ and micronutrient differed significantly with respect to the percent increment in plant height. The treatment $\mathrm{T}_{6}$ chelated micronutrient @ 3.0g/lit was recorded maximum percent increment in plant height (3.23) and was at par with treatment $\mathrm{T}_{5}$ (3.03) however minimum percent increment in plant height (1.98) was recorded in treatment $T_{1}$ chelated calcium @0.5g/lt.

The data presented in table 1 indicated that, method of application of chelated $\mathrm{Ca}$ and micronutrient significantly affected with respect to the increment in height of plant. The maximum percent increment in plant height (3.35) was recorded in method $\mathrm{M}_{3}$ i.e. foliar + soil application method of application followed by the method $\mathrm{M}_{1}$ i.e. foliar method of application 2.72. However minimum percent increment in plant height (1.45) was recorded in method $\mathrm{M}_{2}$ i.e. soil method of application.

The data presented in table 1 indicated that, interaction effect of chelated $\mathrm{Ca}$, micronutrient and methods of application was found to be significantly affected with respect to the percent increment in height of the plant. However, maximum percent increment in plant height (3.87) was recorded in treatment combination $\mathrm{T}_{6} \mathrm{M}_{3}$ i.e. chelated micronutrient @ 3.0g/lit by foliar + soil method of application. The treatment combination $\mathrm{T}_{5} \mathrm{M}_{3}$ (3.67) and $\mathrm{T}_{5} \mathrm{M}_{1}$ (3.50) were at par with the treatment combination $\mathrm{T}_{6} \mathrm{M}_{3}$. However minimum percent increment in plant height (1.28) was recorded in control.

\section{Number of branches per plant}

Data on percent increment in number of branches of pomegranate were significantly influenced by different concentration of chelated $\mathrm{Ca}$ and micronutrient and methods of application as presented in table 2 .

The data presented in table 2 indicated that, different concentration of chelated $\mathrm{Ca}$ and micronutrient differed significantly with respect to the percent increment in number of branches of pomegranate. The treatment $\mathrm{T}_{6}$ i.e. chelated micronutrient @ 3.0g/lit recorded maximum percent increment in number of branches $(9.51)$ followed by the treatment $\mathrm{T}_{5}$ (7.58) and $\mathrm{T}_{4}$ (7.05). Whereas minimum percent increment in number of branches (4.69) was recorded in treatment $\mathrm{T}_{2}$ chelated calcium@1.0\%.

The data presented in table 2 indicated that, method of application of chelated $\mathrm{Ca}$ and micronutrient significantly affected the percent increment in number of branches of pomegranate. The maximum percent increment in number of branches (8.47) was recorded in $\mathrm{M}_{3}$ foliar + soil method of application followed by treatment i.e. $\mathrm{M}_{1}$ foliar method of application 7.82. However minimum percent increment in number of branches was recorded (2.58) in treatment $\mathrm{M}_{2}$ i.e. soil method of application.

The data presented in table 2 indicated that, interaction effect of chelated $\mathrm{Ca}$ and micronutrient and methods of application was found to be significant with respect to the percent increment in number of branches of pomegranate. However maximum percent increment in number of branches (18.66) was recorded in treatment combination $\mathrm{T}_{6} \mathrm{M}_{3}$ i.e. chelated micronutrient @ 3.0g/lit by foliar + 
soil method of application followed by the treatment combination $\mathrm{T}_{5} \mathrm{M}_{3} \quad$ (11.11). However minimum percent increment in number of branches (1.09) was recorded in treatment combination $\mathrm{T}_{1} \mathrm{M}_{2}$ i.e. chelated $\mathrm{Ca}$ @ $0.5 \mathrm{~g} /$ lit by soil method of application.

\section{Number of leaves}

Data on percent increment in number of leaves of pomegranate was significantly influenced by different treatments of chelated calcium and micronutrient and methods of application as presented in table 3 .

The data presented in table 3 indicated that, different concentration of chelated $\mathrm{Ca}$ and micronutrient differed significantly with respect to the percent increment in number of leaves of pomegranate. The treatment $\mathrm{T}_{6}$ i.e. chelated micronutrient @ 3.0g/lit was recorded significantly maximum increment in number of leaves (23.22) followed by the treatment $\mathrm{T}_{5}$ (17.74) and $\mathrm{T}_{4}$ (11.22). However significantly minimum increment in number of leaves of pomegranate (6.21) was recorded in treatment $\mathrm{T}_{1}$ i.e. chelated calcium @ $0.5 \mathrm{~g} / \mathrm{lit}$.

The data presented in table 3 indicated that, method of application of chelated $\mathrm{Ca}$ and micronutrient affected significantly the increment in number of leaves of pomegranate. The maximum increment in number of leaves (18.64) was recorded in $\mathrm{M}_{3}$ i.e. foliar + soil method of application followed by treatment $\mathrm{M}_{1}$ i.e. foliar method of application (11.99). However minimum increment in number of leaves was recorded (4.60) in $\mathrm{M}_{2}$ i.e. soil method of application.

The data presented in table 3 indicated that, interaction effect of chelated $\mathrm{Ca}$ and micronutrient and methods of application was found to be differed significantly with respect to the percent increment in number of leaves of pomegranate. However significantly maximum increment in number of leaves (28.99) was recorded in treatment combination $\mathrm{T}_{6} \mathrm{M}_{3}$ i.e. chelated micronutrient @ 3.0 g/lit by foliar + soil method of application followed by the treatment combination $\mathrm{T}_{5} \mathrm{M}_{3}$ (26.71) and $\mathrm{T}_{6} \mathrm{M}_{1}$ (26.20). However minimum percent increment in number of leaves of pomegranate (1.09) was recorded in control.

\section{Plant spread}

The spread of plant of pomegranate were nonsignificantly affected by different concentration of chelated $\mathrm{Ca}$, micronutrient and methods of application. The data related to spread of plant in $\mathrm{N}-\mathrm{S}$ direction presented in table 5 .

The spread of plant of pomegranate were significantly affected by different treatments of chelated $\mathrm{Ca}$, micronutrient and methods of application. The data related to spread of plant in E-W direction presented in table 5.

Data presented in table 5 indicated that, the chelated $\mathrm{Ca}$ and micronutrient application significantly affected the spread of plant in E$\mathrm{W}$ direction. The treatment $\mathrm{T}_{6}$ i.e. chelated micronutrient@3.0 g/lit recorded maximum spread of plant in E-W direction $225.00 \mathrm{~cm}$. and $\mathrm{T}_{4}$ i.e. chelated micronutrient@2.0 g/lit $223.33 \mathrm{~cm}$ and $T_{5}$ i.e. chelated micronutrient @ $2.5 \mathrm{~g} /$ lit 220.33 were at par with the highest value. The minimum spread of plant was recorded $191.08 \mathrm{~cm}$ in $\mathrm{T}_{2}$ i.e. chelated $\mathrm{Ca}$ @ $1.0 \mathrm{~g} / \mathrm{lit}$.

Data presented in table 5 indicated that, the methods of application also significantly affected the spread of plant in E-W direction of pomegranate. The method $\mathrm{M}_{1}$ i.e. foliar application recorded maximum spread of plant $220.37 \mathrm{~cm}$ followed by $\mathrm{M}_{3}$ i.e. foliar + soil application $212.75 \mathrm{~cm}$. The minimum spread of plant was recorded $205.54 \mathrm{~cm}$ in $\mathrm{M}_{2}$ i.e. soil method of application. 
Table.1 Effect of different concentration and methods of application of chelated $\mathrm{Ca}$ and micronutrient on height of plant

\begin{tabular}{|c|c|c|c|c|}
\hline $\begin{array}{c}\text { Treatment } \\
\text { No. }\end{array}$ & Treatment & $\begin{array}{l}\text { Height of plant } \\
\text { (cm.) before } \\
\text { spraying }\end{array}$ & $\begin{array}{l}\text { Height of plant } \\
\text { (cm.) after } \\
\text { spraying }\end{array}$ & $\begin{array}{c}\text { Percent } \\
\text { increment in } \\
\text { height of plant }\end{array}$ \\
\hline \multicolumn{5}{|c|}{ Factor A: Concentration of chelated $\mathrm{Ca}$ and micronutrient } \\
\hline 1 & $\mathrm{~T}_{1}$ & 205.92 & 210.00 & $1.98(1.16)$ \\
\hline 2 & $\mathrm{~T}_{2}$ & 156.42 & 160.33 & $2.49(1.45)$ \\
\hline 3 & $T_{3}$ & 197.00 & 201.17 & $2.11(1.23)$ \\
\hline 4 & $\mathrm{~T}_{4}$ & 200.17 & 205.58 & $2.70(1.56)$ \\
\hline 5 & $\mathrm{~T}_{5}$ & 188.17 & 193.88 & $3.03(1.73)$ \\
\hline 6 & $\mathrm{~T}_{6}$ & 181.25 & 187.12 & $3.23(1.84)$ \\
\hline \multicolumn{2}{|l|}{ SE \pm} & 4.10 & 4.28 & 0.08 \\
\hline CD at $5 \%$ & & 12.23 & 12.75 & 0.20 \\
\hline \multicolumn{5}{|c|}{ Factor M: Methods of application } \\
\hline 1 & $\mathrm{M}_{1}$ & 190.00 & 195.17 & $2.72(1.59)$ \\
\hline 2 & $\mathrm{M}_{2}$ & 189.42 & 192.71 & $1.45(1.00)$ \\
\hline 3 & $\mathrm{M}_{3}$ & 184.96 & 191.17 & $3.35(1.94)$ \\
\hline \multicolumn{2}{|l|}{ SE \pm} & 2.90 & 3.02 & 0.06 \\
\hline CD at 5\% & & 8.65 & 9.01 & 0.18 \\
\hline \multicolumn{5}{|c|}{ Interaction effect ( $\mathrm{T} \times \mathrm{M})$} \\
\hline $\mathrm{T}_{1}$ & $\mathrm{~T}_{1} \mathrm{M}_{1}$ & 199.00 & 203.00 & $2.01(1.13)$ \\
\hline $\mathrm{T}_{2}$ & $\mathrm{~T}_{1} \mathrm{M}_{2}$ & 205.50 & 208.25 & $1.33(0.75)$ \\
\hline $\mathrm{T}_{3}$ & $\mathrm{~T}_{1} \mathrm{M}_{3}$ & 213.25 & 218.75 & $2.34(1.60)$ \\
\hline $\mathrm{T}_{4}$ & $\mathrm{~T}_{2} \mathrm{M}_{1}$ & 154.75 & 158.50 & $2.42(1.44)$ \\
\hline $\mathrm{T}_{5}$ & $\mathrm{~T}_{2} \mathrm{M}_{2}$ & 162.50 & 165.00 & $1.53(0.90)$ \\
\hline $\mathrm{T}_{6}$ & $\mathrm{~T}_{2} \mathrm{M}_{3}$ & 152.00 & 157.50 & $3.61(1.98)$ \\
\hline $\mathrm{T}_{7}$ & $\mathrm{~T}_{3} \mathrm{M}_{1}$ & 203.50 & 207.25 & $1.84(1.12)$ \\
\hline $\mathrm{T}_{8}$ & $\mathrm{~T}_{3} \mathrm{M}_{2}$ & 195.50 & 198.00 & $1.27(0.7)$ \\
\hline $\mathrm{T}_{9}$ & $\mathrm{~T}_{3} \mathrm{M}_{3}$ & 192.00 & 198.25 & $3.25(1.87)$ \\
\hline $\mathrm{T}_{10}$ & $\mathrm{~T}_{4} \mathrm{M}_{1}$ & 202.25 & 208.25 & $2.96(1.69)$ \\
\hline $\mathrm{T}_{11}$ & $\mathrm{~T}_{4} \mathrm{M}_{2}$ & 210.00 & 214.00 & $1.90(1.09)$ \\
\hline $\mathrm{T}_{12}$ & $\mathrm{~T}_{4} \mathrm{M}_{3}$ & 188.25 & 194.50 & $3.32(1.89)$ \\
\hline $\mathrm{T}_{13}$ & $\mathrm{~T}_{5} \mathrm{M}_{1}$ & 200.00 & 207.00 & 3.50 (1.99) \\
\hline $\mathrm{T}_{14}$ & $\mathrm{~T}_{5} \mathrm{M}_{2}$ & 187.50 & 191.12 & $1.93(1.09)$ \\
\hline $\mathrm{T}_{15}$ & $\mathrm{~T}_{5} \mathrm{M}_{3}$ & 177.00 & 183.50 & $3.67(2.10)$ \\
\hline $\mathrm{T}_{16}$ & $\mathrm{~T}_{6} \mathrm{M}_{1}$ & 181.00 & 187.00 & $3.31(1.90)$ \\
\hline $\mathrm{T}_{17}$ & $\mathrm{~T}_{6} \mathrm{M}_{2}$ & 175.50 & 179.88 & $2.49(1.42)$ \\
\hline \multirow[t]{2}{*}{$\mathrm{T}_{18}$} & $\mathrm{~T}_{6} \mathrm{M}_{3}$ & 187.25 & 194.50 & $3.87(2.21)$ \\
\hline & $\mathrm{T}_{0} \mathrm{M}_{0}$ & 194.75 & 197.25 & $1.28(1.29)$ \\
\hline \multicolumn{2}{|l|}{$\mathrm{SE}_{ \pm}$} & & & 0.1 \\
\hline \multicolumn{2}{|l|}{ CD at $5 \%$} & & & 0.45 \\
\hline
\end{tabular}


Table.2 Effect of different concentration and methods of application of chelated $\mathrm{Ca}$ and micronutrient on number of branches

\begin{tabular}{|c|c|c|c|c|}
\hline $\begin{array}{c}\text { Treatment } \\
\text { No. }\end{array}$ & Treatment & $\begin{array}{c}\text { Number of } \\
\text { branches } \\
\text { (before } \\
\text { spraying) }\end{array}$ & $\begin{array}{c}\text { Number of } \\
\text { branches (after } \\
\text { spraying) }\end{array}$ & $\begin{array}{c}\text { Percent } \\
\text { increment in } \\
\text { number of } \\
\text { branches }\end{array}$ \\
\hline \multicolumn{5}{|c|}{ Factor T: Concentration of chelated $\mathrm{Ca}$ and Micronutrient } \\
\hline 1 & $\mathrm{~T}_{1}$ & 10.58 & 11.12 & $5.10(2.21)$ \\
\hline 2 & $\mathrm{~T}_{2}$ & 10.66 & 11.16 & $4.69(2.38)$ \\
\hline 3 & $\mathrm{~T}_{3}$ & 10.50 & 11.08 & $5.52(3.02)$ \\
\hline 4 & $\mathrm{~T}_{4}$ & 9.50 & 10.17 & $7.05(3.76)$ \\
\hline 5 & $\mathrm{~T}_{5}$ & 8.83 & 9.50 & $7.58(4.10)$ \\
\hline 6 & $\mathrm{~T}_{6}$ & 7.25 & 7.94 & $9.51(3.51)$ \\
\hline \multicolumn{2}{|l|}{$\mathrm{SE}_{ \pm}$} & 0.19 & 0.18 & 0.12 \\
\hline CD at $5 \%$ & & 0.57 & 0.54 & 0.36 \\
\hline \multicolumn{5}{|c|}{ Factor M: Methods of Application } \\
\hline 1 & $\mathrm{M}_{1}$ & 9.58 & 10.33 & $7.82(3.54)$ \\
\hline 2 & $\mathrm{M}_{2}$ & 9.29 & 9.53 & $2.58(1.53)$ \\
\hline 3 & $\mathrm{M}_{3}$ & 9.79 & 10.62 & $8.47(4.42)$ \\
\hline \multicolumn{2}{|l|}{ SE \pm} & 0.13 & 0.13 & 0.08 \\
\hline CD at $5 \%$ & & 0.40 & 0.38 & 0.25 \\
\hline \multicolumn{5}{|c|}{ Interaction effect ( $\mathrm{T} \times \mathrm{M})$} \\
\hline $\mathrm{T}_{1}$ & $\mathrm{~T}_{1} \mathrm{M}_{1}$ & 10.00 & 10.50 & $5.00(2.92)$ \\
\hline $\mathrm{T}_{2}$ & $\mathrm{~T}_{1} \mathrm{M}_{2}$ & 11.00 & 11.12 & $3.12(0.68)$ \\
\hline$T_{3}$ & $\mathrm{~T}_{1} \mathrm{M}_{3}$ & 10.75 & 11.75 & $9.30(3.04)$ \\
\hline $\mathrm{T}_{4}$ & $\mathrm{~T}_{2} \mathrm{M}_{1}$ & 10.50 & 11.00 & $4.76(2.29)$ \\
\hline $\mathrm{T}_{5}$ & $\mathrm{~T}_{2} \mathrm{M}_{2}$ & 10.25 & 10.37 & $1.17(0.57)$ \\
\hline $\mathrm{T}_{6}$ & $\mathrm{~T}_{2} \mathrm{M}_{3}$ & 11.25 & 12.12 & $7.73(4.30)$ \\
\hline $\mathrm{T}_{7}$ & $\mathrm{~T}_{3} \mathrm{M}_{1}$ & 11.75 & 12.62 & $7.40(4.12)$ \\
\hline $\mathrm{T}_{8}$ & $\mathrm{~T}_{3} \mathrm{M}_{2}$ & 10.00 & 10.25 & $2.50(1.30)$ \\
\hline $\mathrm{T}_{9}$ & $\mathrm{~T}_{3} \mathrm{M}_{3}$ & 9.75 & 10.37 & $6.35(3.63)$ \\
\hline $\mathrm{T}_{10}$ & $\mathrm{~T}_{4} \mathrm{M}_{1}$ & 9.75 & 10.50 & $7.69(4.29)$ \\
\hline $\mathrm{T}_{11}$ & $\mathrm{~T}_{4} \mathrm{M}_{2}$ & 8.50 & 8.87 & $6.06(2.47)$ \\
\hline$T_{12}$ & $\mathrm{~T}_{4} \mathrm{M}_{3}$ & 10.25 & 11.15 & $8.78(4.51)$ \\
\hline$T_{13}$ & $\mathrm{~T}_{5} \mathrm{M}_{1}$ & 9.50 & 10.25 & $7.89(4.30)$ \\
\hline $\mathrm{T}_{14}$ & $\mathrm{~T}_{5} \mathrm{M}_{2}$ & 8.00 & 8.25 & $4.00(1.90)$ \\
\hline$T_{15}$ & $\mathrm{~T}_{5} \mathrm{M}_{3}$ & 9.00 & 10.00 & $11.11(4.93)$ \\
\hline$T_{16}$ & $\mathrm{~T}_{6} \mathrm{M}_{1}$ & 6.00 & 7.12 & $8.00(3.30)$ \\
\hline$T_{17}$ & $\mathrm{~T}_{6} \mathrm{M}_{2}$ & 8.00 & 8.32 & $4.35(2.21)$ \\
\hline \multirow[t]{2}{*}{$\mathrm{T}_{18}$} & $\mathrm{~T}_{6} \mathrm{M}_{3}$ & 7.75 & 8.37 & $18.66(6.09)$ \\
\hline & $\mathrm{T}_{0} \mathrm{M}_{0}$ & 8.25 & 8.30 & $1.09(0.66)$ \\
\hline \multicolumn{2}{|l|}{$\mathrm{SE} \pm$} & & & 0.21 \\
\hline \multicolumn{2}{|l|}{ CD at 5\% } & & & 0.63 \\
\hline
\end{tabular}


Table.3 Effect of different concentration and methods of application of chelated $\mathrm{Ca}$, micronutrient on number of leaves

\begin{tabular}{|c|c|c|c|c|}
\hline $\begin{array}{c}\text { Treatment } \\
\text { No. }\end{array}$ & Treatment & $\begin{array}{l}\text { Number of } \\
\text { leaves (before } \\
\text { application) }\end{array}$ & $\begin{array}{l}\text { Number of } \\
\text { leaves (after } \\
\text { application) }\end{array}$ & $\begin{array}{c}\text { Percent } \\
\text { increment in } \\
\text { number of } \\
\text { leaves }\end{array}$ \\
\hline \multicolumn{5}{|c|}{ Factor T: Concentration of chelated $\mathrm{Ca}$ and Micronutrient } \\
\hline 1 & $\mathrm{~T}_{1}$ & 794.00 & 843.33 & $6.21(3.68)$ \\
\hline 2 & $\mathrm{~T}_{2}$ & 1048.80 & 1124.70 & $7.23(4.48)$ \\
\hline 3 & $T_{3}$ & 881.67 & 965.93 & $9.55(5.83)$ \\
\hline 4 & $\mathrm{~T}_{4}$ & 977.00 & 1086.70 & $11.22(6.60)$ \\
\hline 5 & $\mathrm{~T}_{5}$ & 888.08 & 1045.70 & $17.74(10.51)$ \\
\hline 6 & $\mathrm{~T}_{6}$ & 538.50 & 663.58 & $23.22(12.43)$ \\
\hline \multicolumn{2}{|l|}{$\mathbf{S E} \pm$} & 122.56 & 16.05 & 0.24 \\
\hline \multirow{2}{*}{\multicolumn{5}{|c|}{\begin{tabular}{l|l} 
CD at 5\% \\
Factor M: Methods of Application
\end{tabular}}} \\
\hline & & & & \\
\hline 1 & $\mathrm{M}_{1}$ & 944.04 & 1057.30 & $11.99(7.88)$ \\
\hline 2 & $\mathrm{M}_{2}$ & 816.58 & 854.21 & $4.60(3.04)$ \\
\hline 3 & $\mathrm{M}_{3}$ & 803.62 & 953.42 & $18.64(10.85)$ \\
\hline \multicolumn{2}{|l|}{$\overline{\mathbf{S E} \pm}$} & 15.95 & 11.35 & 0.17 \\
\hline \multirow{2}{*}{\multicolumn{3}{|c|}{$\begin{array}{l}\text { CD at 5\% } \\
\text { Interaction Effect }(\mathrm{T} \times \mathrm{M})\end{array}$}} & 33.81 & 0.50 \\
\hline & & & \\
\hline $\mathrm{T}_{1}$ & $\mathrm{~T}_{1} \mathrm{M}_{1}$ & 569.00 & 620.00 & $8.96(5.07)$ \\
\hline $\mathrm{T}_{2}$ & $\mathrm{~T}_{1} \mathrm{M}_{2}$ & 657.50 & 676.00 & $2.85(2.73)$ \\
\hline $\mathrm{T}_{3}$ & $\mathrm{~T}_{1} \mathrm{M}_{3}$ & 945.00 & 1025.00 & $8.46(4.77)$ \\
\hline $\mathrm{T}_{4}$ & $\mathrm{~T}_{2} \mathrm{M}_{1}$ & 1204.00 & 1279.00 & $6.22(4.63)$ \\
\hline $\mathrm{T}_{5}$ & $\mathrm{~T}_{2} \mathrm{M}_{2}$ & 1070.00 & 1097.50 & $2.57(1.63)$ \\
\hline $\mathrm{T}_{6}$ & $\mathrm{~T}_{2} \mathrm{M}_{3}$ & 872.50 & 997.50 & $14.32(7.35)$ \\
\hline $\mathrm{T}_{7}$ & $\mathrm{~T}_{3} \mathrm{M}_{1}$ & 1071.20 & 1181.80 & $10.27(6.83)$ \\
\hline $\mathrm{T}_{8}$ & $\mathrm{~T}_{3} \mathrm{M}_{2}$ & 922.50 & 959.50 & $4.01(2.24)$ \\
\hline $\mathrm{T}_{9}$ & $\mathrm{~T}_{3} \mathrm{M}_{3}$ & 651.25 & 756.50 & $16.16(8.42)$ \\
\hline $\mathrm{T}_{10}$ & $\mathrm{~T}_{4} \mathrm{M}_{1}$ & 1127.50 & 1229.50 & $9.04(5.15)$ \\
\hline $\mathrm{T}_{11}$ & $\mathrm{~T}_{4} \mathrm{M}_{2}$ & 873.50 & 905.50 & $3.66(2.30)$ \\
\hline $\mathrm{T}_{12}$ & $\mathrm{~T}_{4} \mathrm{M}_{3}$ & 930.00 & 1125.00 & $20.96(12.34)$ \\
\hline$T_{13}$ & $\mathrm{~T}_{5} \mathrm{M}_{1}$ & 1094.20 & 1278.00 & $16.79(10.94)$ \\
\hline $\mathrm{T}_{14}$ & $\mathrm{~T}_{5} \mathrm{M}_{2}$ & 732.50 & 797.75 & $8.90(5.14)$ \\
\hline $\mathrm{T}_{15}$ & $\mathrm{~T}_{5} \mathrm{M}_{3}$ & 837.50 & 1061.20 & $26.71(15.45)$ \\
\hline$T_{16}$ & $\mathrm{~T}_{6} \mathrm{M}_{1}$ & 598.25 & 755.00 & $26.20(14.79)$ \\
\hline$T_{17}$ & $\mathrm{~T}_{6} \mathrm{M}_{2}$ & 433.00 & 480.00 & $10.85(5.72)$ \\
\hline \multirow[t]{2}{*}{$\mathrm{T}_{18}$} & $\mathrm{~T}_{6} \mathrm{M}_{3}$ & 585.50 & 755.25 & $28.99(16.78)$ \\
\hline & $\mathrm{T}_{0} \mathrm{M}_{0}$ & 868.00 & 885.00 & $1.95(1.20)$ \\
\hline \multicolumn{2}{|l|}{$\mathrm{SE}_{ \pm}$} & & & 0.41 \\
\hline \multicolumn{2}{|l|}{ CD at $5 \%$} & & & 1.24 \\
\hline
\end{tabular}


Table.4 Effect of different concentration and methods of application of chelated $\mathrm{Ca}$ and micronutrient on girth of stem

\begin{tabular}{|c|c|c|}
\hline $\begin{array}{l}\text { Treatment } \\
\text { No. }\end{array}$ & Treatment & Girth of stem $(\mathbf{c m}$.) \\
\hline \multicolumn{3}{|c|}{ Factor T: Concentration of chelated Ca and Micronutrient } \\
\hline 1 & $\mathrm{~T}_{1}$ & 14.19 \\
\hline 2 & $\mathrm{~T}_{2}$ & 17.06 \\
\hline 3 & $\mathrm{~T}_{3}$ & 17.67 \\
\hline 4 & $\mathrm{~T}_{4}$ & 18.51 \\
\hline 5 & $\mathrm{~T}_{5}$ & 19.08 \\
\hline 6 & $\mathrm{~T}_{6}$ & 20.05 \\
\hline \multicolumn{2}{|l|}{ SE \pm} & 0.07 \\
\hline \multirow{2}{*}{\multicolumn{3}{|c|}{$\begin{array}{l}\text { CD at 5\% } \\
\text { Factor M: Methods of Application }\end{array}$}} \\
\hline & & \\
\hline 1 & $\mathrm{M}_{1}$ & 17.78 \\
\hline 2 & $\mathrm{M}_{2}$ & 17.37 \\
\hline 3 & $\mathrm{M}_{3}$ & 18.13 \\
\hline \multicolumn{2}{|l|}{$\mathrm{SE}_{ \pm}$} & 0.05 \\
\hline CD at $5 \%$ & & 0.15 \\
\hline \multicolumn{3}{|c|}{ Interaction effect ( $\mathbf{T} \times \mathbf{M})$} \\
\hline $\mathrm{T}_{1}$ & $\mathrm{~T}_{1} \mathrm{M}_{1}$ & 15.26 \\
\hline $\mathrm{T}_{2}$ & $\mathrm{~T}_{1} \mathrm{M}_{2}$ & 16.12 \\
\hline $\mathrm{T}_{3}$ & $\mathrm{~T}_{1} \mathrm{M}_{3}$ & 19.49 \\
\hline $\mathrm{T}_{4}$ & $\mathrm{~T}_{2} \mathrm{M}_{1}$ & 17.40 \\
\hline$T_{5}$ & $\mathrm{~T}_{2} \mathrm{M}_{2}$ & 15.31 \\
\hline $\mathrm{T}_{6}$ & $\mathrm{~T}_{2} \mathrm{M}_{3}$ & 17.80 \\
\hline $\mathrm{T}_{7}$ & $\mathrm{~T}_{3} \mathrm{M}_{1}$ & 16.15 \\
\hline $\mathrm{T}_{8}$ & $\mathrm{~T}_{3} \mathrm{M}_{2}$ & 17.23 \\
\hline $\mathrm{T}_{9}$ & $\mathrm{~T}_{3} \mathrm{M}_{3}$ & 18.2 \\
\hline $\mathrm{T}_{10}$ & $\mathrm{~T}_{4} \mathrm{M}_{1}$ & 20.54 \\
\hline $\mathrm{T}_{11}$ & $\mathrm{~T}_{4} \mathrm{M}_{2}$ & 15.29 \\
\hline$T_{12}$ & $\mathrm{~T}_{4} \mathrm{M}_{3}$ & 20.51 \\
\hline $\mathrm{T}_{13}$ & $\mathrm{~T}_{5} \mathrm{M}_{1}$ & 18.16 \\
\hline $\mathrm{T}_{14}$ & $\mathrm{~T}_{5} \mathrm{M}_{2}$ & 16.79 \\
\hline $\mathrm{T}_{15}$ & $\mathrm{~T}_{5} \mathrm{M}_{3}$ & 20.58 \\
\hline $\mathrm{T}_{16}$ & $\mathrm{~T}_{6} \mathrm{M}_{1}$ & 18.88 \\
\hline$T_{17}$ & $\mathrm{~T}_{6} \mathrm{M}_{2}$ & 16.42 \\
\hline \multirow[t]{2}{*}{$\mathrm{T}_{18}$} & $\mathrm{~T}_{6} \mathrm{M}_{3}$ & 22.23 \\
\hline & $\mathrm{T}_{0} \mathrm{M}_{0}$ & 11.13 \\
\hline \multicolumn{2}{|l|}{$\mathrm{SE}_{ \pm}$} & 0.13 \\
\hline \multicolumn{2}{|l|}{ CD at $5 \%$} & 0.39 \\
\hline
\end{tabular}


Table.5 Effect of different concentration and methods of application of chelated $\mathrm{Ca}$ and micronutrient on Spread of plant (cm.)

\begin{tabular}{|c|c|c|c|}
\hline $\begin{array}{c}\text { Treatment } \\
\text { No. }\end{array}$ & Treatment & $\begin{array}{l}\text { Spread of plant in } \\
\text { N-S direction }\end{array}$ & $\begin{array}{l}\text { Spread of plan in } \\
\text { E-W direction }\end{array}$ \\
\hline \multicolumn{4}{|c|}{ Factor T: Concentration of chelated $\mathrm{Ca}$ and Micronutrient } \\
\hline 1 & $\mathrm{~T}_{1}$ & 204.17 & 205.50 \\
\hline 2 & $\mathrm{~T}_{2}$ & 228.83 & 191.08 \\
\hline 3 & $T_{3}$ & 220.58 & 212.08 \\
\hline 4 & $\mathrm{~T}_{4}$ & 215.75 & 223.33 \\
\hline 5 & $\mathrm{~T}_{5}$ & 232.52 & 220.33 \\
\hline 6 & $\mathrm{~T}_{6}$ & 235.92 & 225.00 \\
\hline \multicolumn{2}{|l|}{$\mathbf{S E}+$} & 12.99 & 1.69 \\
\hline \multirow{2}{*}{\multicolumn{3}{|c|}{$\begin{array}{l}\text { CD at 5\% } \\
\text { Factor M: Methods of Application }\end{array}$}} & 5.04 \\
\hline & & & \\
\hline 1 & $\mathrm{M}_{1}$ & 231.12 & 212.75 \\
\hline 2 & $\mathrm{M}_{2}$ & 216.63 & 205.54 \\
\hline 3 & $\mathrm{M}_{3}$ & 221.12 & 220.37 \\
\hline \multicolumn{2}{|l|}{$\mathrm{SE}_{ \pm}$} & 9.19 & 1.20 \\
\hline CD at $5 \%$ & & NS & 3.56 \\
\hline \multicolumn{3}{|c|}{ Interaction Effect (T x M) } & \\
\hline $\mathrm{T}_{1}$ & $\mathrm{~T}_{1} \mathrm{M}_{1}$ & 202.50 & 187.50 \\
\hline $\mathrm{T}_{2}$ & $\mathrm{~T}_{1} \mathrm{M}_{2}$ & 217.50 & 123.75 \\
\hline $\mathrm{T}_{3}$ & $\mathrm{~T}_{1} \mathrm{M}_{3}$ & 227.25 & 220.25 \\
\hline $\mathrm{T}_{4}$ & $\mathrm{~T}_{2} \mathrm{M}_{1}$ & 231.25 & 213.75 \\
\hline $\mathrm{T}_{5}$ & $\mathrm{~T}_{2} \mathrm{M}_{2}$ & 225.00 & 215.50 \\
\hline $\mathrm{T}_{6}$ & $\mathrm{~T}_{2} \mathrm{M}_{3}$ & 230.25 & 231.75 \\
\hline $\mathrm{T}_{7}$ & $\mathrm{~T}_{3} \mathrm{M}_{1}$ & 233.50 & 223.50 \\
\hline $\mathrm{T}_{8}$ & $\mathrm{~T}_{3} \mathrm{M}_{2}$ & 229.05 & 220.00 \\
\hline $\mathrm{T}_{9}$ & $\mathrm{~T}_{3} \mathrm{M}_{3}$ & 235.00 & 231.50 \\
\hline $\mathrm{T}_{10}$ & $\mathrm{~T}_{4} \mathrm{M}_{1}$ & 243.50 & 240.00 \\
\hline $\mathrm{T}_{11}$ & $\mathrm{~T}_{4} \mathrm{M}_{2}$ & 223.25 & 216.50 \\
\hline $\mathrm{T}_{12}$ & $\mathrm{~T}_{4} \mathrm{M}_{3}$ & 241.00 & 213.50 \\
\hline$T_{13}$ & $\mathrm{~T}_{5} \mathrm{M}_{1}$ & 251.00 & 238.25 \\
\hline $\mathrm{T}_{14}$ & $\mathrm{~T}_{5} \mathrm{M}_{2}$ & 227.00 & 208.75 \\
\hline $\mathrm{T}_{15}$ & $\mathrm{~T}_{5} \mathrm{M}_{3}$ & 258.75 & 211.25 \\
\hline$T_{16}$ & $\mathrm{~T}_{6} \mathrm{M}_{1}$ & 225.00 & 219.25 \\
\hline$T_{17}$ & $\mathrm{~T}_{6} \mathrm{M}_{2}$ & 178.00 & 161.25 \\
\hline \multirow[t]{2}{*}{$\mathrm{T}_{18}$} & $\mathrm{~T}_{6} \mathrm{M}_{3}$ & 274.25 & 255.75 \\
\hline & $\mathrm{T}_{0} \mathrm{M}_{0}$ & 134.50 & 174.75 \\
\hline \multicolumn{2}{|l|}{ SE \pm} & NS & 2.94 \\
\hline \multicolumn{2}{|l|}{ CD at 5\% } & & 11.98 \\
\hline
\end{tabular}




\section{Treatment Details}

\section{First factor}

1. Chelated calcium @ 0.5 gm/lit $\left(\mathrm{T}_{1}\right)$

2. Chelated calcium @1.0 gm/lit $\left(\mathrm{T}_{2}\right)$

3. Chelated calcium @1.5 gm/lit $\left(\mathrm{T}_{3}\right)$

4. Chelated Micronutrient @ 2 gm/lit $\left(\mathrm{T}_{4}\right)$

5. Chelated Micronutrient @2.5 gm/lit $\left(\mathrm{T}_{5}\right)$

6. Chelated Micronutrient @3.0 gm/lit $\left(\mathrm{T}_{6}\right)$

Data presented in table 5 indicated that, the interaction effect of different concentration of chelated $\mathrm{Ca}$ and micronutrient and methods of application was significantly affected the spread of plant in E-W direction of pomegranate. The treatment combination $\mathrm{T}_{6} \mathrm{M}_{3}$ i.e. chelated micronutrient @ $3.0 \mathrm{~g} / \mathrm{lit}$ and foliar + soil method of application recorded maximum spread of plan in E-W direction $255.75 \mathrm{~cm}$, followed by $\mathrm{T}_{4} \mathrm{M}_{1}$ $240.00 \mathrm{~cm}$ and $\mathrm{T}_{5} \mathrm{M}_{1} 238.25 \mathrm{~cm}$. The minimum spread of plant was recorded $123.75 \mathrm{~cm}$ in $\mathrm{T}_{5} \mathrm{M}_{3}$ i.e. chelated micronutrient @ $2.5 \mathrm{~g} / \mathrm{lit}$ by foliar + soil method of application.

\section{Girth of stem}

The stem girth of plant was significantly affected highly by different concentration of chelated $\mathrm{Ca}$ and micronutrient and methods of application. The data related to girth of stem were presented in table 4.

The data presented in table 4 indicated that, application of different concentration of treatment was significantly affected the girth of stem of pomegranate. Maximum girth of stem (20.05) was recorded in treatment $\mathrm{T}_{6}$ i.e. chelated micronutrient @ 3.0g/lit followed by $\mathrm{T}_{5}$ i.e. chelated micronutrient @ $2.5 \mathrm{~g} / \mathrm{lit}$ (19.08) and $\mathrm{T}_{4}$ i.e. chelated micronutrient @ $2.0 \mathrm{~g} / \mathrm{lit}$ (18.51). Whereas significantly minimum girth of stem (14.19) was recorded in treatment $\mathrm{T}_{2}$ i.e. chelated $\mathrm{Ca}$ and micronutrient@0.5g/lit.

\author{
Second Factor \\ 1.Spraying $\left(\mathrm{M}_{1}\right)$ \\ 2.Soil application $\left(\mathrm{M}_{2}\right)$ \\ 3.Spraying+ soil application $\left(\mathrm{M}_{3}\right)$
}

The data presented in table 4 indicated that, method of application of treatment significantly affected highly with respect to the girth of the stem of pomegranate. The maximum girth of stem (18.13) was recorded in $\mathrm{M}_{3}$ i.e. foliar + soil method of application followed by $\mathrm{M}_{1}$ i.e. foliar method of application (17.78). Whereas minimum girth of stem (17.37) was recorded in $\mathrm{M}_{2}$ i.e. soil method of application.

The data presented in table 4 indicated that, Interaction effect of chelated $\mathrm{Ca}$ and micronutrient and methods of application was found to be highly significant with respect to the girth of stem. The maximum girth of stem (22.23) was recorded in treatment combination $\mathrm{T}_{6} \mathrm{M}_{3}$ i.e. chelated micronutrient @ 3.0 g/lit by foliar + soil method of application followed by $\mathrm{T}_{5} \mathrm{M}_{3}$ i.e. chelated micronutrient @ $2.5 \mathrm{~g} / \mathrm{lit}$ by foliar + soil method of application (20.58), $\mathrm{T}_{4} \mathrm{M}_{1}$ chelated micronutrient @ $2.0 \mathrm{~g} /$ lit by foliar method of application (20.54) and $\mathrm{T}_{4} \mathrm{M}_{3}$ i.e. chelated micronutrient @2.0 g/lit by foliar + soil method of application (20.51). Whereas minimum girth of stem (11.13) was recorded in control.

The data presented in table 1 indicated that, different concentration and method of application of chelated $\mathrm{Ca}$, and micronutrient was found to be differed significantly with respect to the percent increment in height of the plant the treatment $\mathrm{T}_{6}$ i.e. chelated micronutrient @ $3.0 \mathrm{~g} / \mathrm{lit}$ is recorded more 
percent increment of plant height (3.23) and at par with treatment $\mathrm{T}_{5}$ chelated micronutrient @2.5 g/lit i.e. (3.03). However minimum percent increment in plant height (1.95) was recorded in treatment $\mathrm{T}_{1}$ i.e. chelated Ca and micronutrient @0.5 g/lit.As regard the method of application the more percent increment in plant height (3.35) was recorded in $\mathrm{M}_{3}$ foliar + soil method of application followed by $\mathrm{M}_{1}$ i.e. foliar method of application 2.72 however less increment in plant height was recorded (1.45) in $\mathrm{M}_{2}$ i.e. soil method of application.

In interaction effect more percent increment in plant height (3.87) was recorded in treatment combination $\mathrm{T}_{6} \mathrm{M}_{3}$ i.e. chelated micronutrient @ 3.0 g/lit by foliar + soil method of application.

This may be due to zinc which plays an important role in the fundamental process involved in the cellular mechanism and respiration. The presence of zinc in chloroplasts, cell was also considered as possible causes of increased growth of plants. Improvement in growth characters due to micronutrient application might be due to enhanced photosynthetic and other metabolic activities related to cell division and elongation as opined by Hatwar et al.,(2003).Similar finding was reported by Veena and Lavania (1998) in Papaya, Bhalerao et al., (2014) in papaya, Meena et al., (2014) in aonla, Jeyabaskaran and Pande (2008) in Banana.

The data presented in table 2 indicated that, different concentration and method of application of chelated $\mathrm{Ca}$, and micronutrient was found to be differed significantly with respect to the percent increment in no. of branches. The treatment $\mathrm{T}_{5}$ i.e. chelated micronutrient @2.5 g/lit recorded maximum increment in no. of branches (9.51). The method of application significantly affected with respect to the percent increment in no. of branches. The more percent increment in no. of branches (8.47) was recorded in $\mathrm{M}_{3}$ i.e. foliar + soil application method of application.

In interaction effect of chelated $\mathrm{Ca}$ and micronutrient and method of application was found to be differed significantly with respect of the percent increment in no. of branches. However more percent increment in no. of branches (18.66) was recorded in treatment combination $\mathrm{T}_{6} \mathrm{M}_{3}$ i.e. chelated micronutrient @ $2.5 \mathrm{~g} /$ lit by foliar + soil method of application however less percent increment in number of branches (1.09) was recorded in control.

Improvement in growth characters with the application of micronutrient might be due to enhanced photosynthetic and other metabolic activities related to cell division and elongation as opined by Hatwar et al., (2003).

The data presented in table 3 indicated that, different concentration and method of application of chelated $\mathrm{Ca}$, and micronutrient was found to be differed significantly with respect to the percent increment in number of leaves. The treatment $\mathrm{T}_{6}$ i.e. chelated micronutrient @ $3.0 \mathrm{~g} / \mathrm{lit}$ recorded maximum increment in no. of leaves (23.22).

The Method of application also significantly influenced the percent increment in number of leaves. The more increment in no. of leaves was recorded in $\mathrm{M}_{3}$ i.e. foliar+ soil method of application (18.64). However more increment in no. of leaves (28.99) was recorded in treatment combination $\mathrm{T}_{6} \mathrm{M}_{3}$ i.e. chelated micronutrient@3.0 g/lit by foliar + soil method of application.

The foliar spray of micronutrients viz. $\mathrm{Mg}$ and $\mathrm{Zn}$ might have induced the synthesis of chlorophyll and thus lead to increase in 
chlorophyll content which in turn resulted in higher vegetative growth parameters. This may be due to zinc which plays an important role in the fundamental process involved in the cellular mechanism and respiration. The presence of zinc in chloroplasts, cell was also considered as possible causes of increased growth of plants.

The results obtained in present study are in agreement with the findings reported by Singh and Rajput (1976), Singaram et al., (2001),Yadav et al., (2010, Balakrishnan (2000) in guava. Bagali et al., (1993) in guava.

The data furnished in table 4 revealed that the $\mathrm{T}_{6}$ i.e. chelated micronutrient @3.0 g/lit recorded maximum girth of stem than other treatments. The $\mathrm{M}_{3}$ i.e. foliar + soil method of application recorded maximum girth of stem. In interaction effect the treatment combination $\mathrm{T}_{18} \quad\left(\mathrm{~T}_{6} \mathrm{M}_{3}\right)$ i.e. chelated micronutrient @ 3.0 g/lit by foliar + soil method of application It may due to accumulation of food material taken by trees through foliage and all micronutrients available in grades. The similar result had been reported by Jitendra kumar, et al., (2015) in guava and Yadav et al., (2010) in banana.

The data furnished in table 5 revealed that the spread of plant in N-S direction non significantly influenced by application of different concentration and method of application of chelated $\mathrm{Ca}$ and micronutrient.

In E-W direction, the treatment $\mathrm{T}_{6}$ i.e. chelated micronutrient @ 3.0 g/lit was recorded maximum spread of plant in E-W direction while minimum spread of plant in E$\mathrm{W}$ direction was recorded in treatment $\mathrm{T}_{1}$ i.e. chelated calcium @ 0.5 g/lit.The foliar method of application was recorded maximum spread of plant in E-W direction. In interaction effect of chelated $\mathrm{Ca}$ and micronutrient and methods of application the data indicated that, the maximum spread of plant in N-S and E-W direction was recorded in $\mathrm{T}_{6} \mathrm{M}_{3}$ i.e. chelated micronutrient @ $3.0 \mathrm{~g} / \mathrm{lit}$ by foliar + soil method of application. While minimum spread of plant in N-S and E-W direction was recorded in control.

It may due to accumulation of food material taken by trees through foliage and soil and all micronutrients available in grades. Zinc plays an important role in the fundamental process involved in the cellular mechanism and respiration. The presence of zinc in chloroplasts, cell was also considered as possible causes of increased growth of plants. These results are in conformity with the findings of Meena et al., (2014) in aonla and Singh and Rajput (1976) in mango these finding similar with above result.

\section{References}

Bagali, A. N., Hulamani, N. C. and G.S. Sulikeri (1993) Effect of foliar application of $\mathrm{Zn}, \mathrm{Mg}$ and $\mathrm{B}$ on growth and yield of guava (Psidium guajava L.) cv. Sardar. Karnataka Journal Agric. Sci., 6(2): (137-141).

Balakrishnan, K. (2000) Foliar spray of zinc, iron, boron and magnesium on vegetative growth, yield and quality of guava. Ann. Plant Physiol. 14 (2): 151153.

Bhalerao, P. P., Patil, B. N., S. J. and S.S.Gaikwad (2014) Effect of foliar application of $\mathrm{Ca}, \mathrm{Zn}, \mathrm{Fe}$ and $\mathrm{B}$ on growth, yield and quality of papaya (Carica papaya) cv. Taiwan Red Lady. Current Horticulture, 2(2): 35-39.

Hatwar G.P., Gonddane S.U., Urkude S.M., Gahukar O.V.(2003) Effect of micronutrients on growth and yield of chilli. Soils and crops. 13:123-125.

Jeyabaskaran, K. J. and Pandey, S. D. (2008) 
Effect of foliar spray of micronutrients in banana under high soil $\mathrm{pH}$ condition. Indian journal Hort. 65(1):102-105.

Kumar J., Kumar R., Ratna, R., Mishra, D. S. (2015). Response of 'Pant Prabhat' guava trees to foliar sprays of zinc, boron, calcium and potassium at different plant growth stages. The Boiscan 10 (2): 495-498.

Lye, C. (2008) Preliminary assessment of the potential for an Australian industry. Rural industries research and development corporation of Australian government. RIRDC publication no. 08/153.p.17.

Malewar, G.U. (1977). Micronurients status, their distribution and availability in soils of Marathwada region of Maharashtra state in relation to soil types and cropping patterns. $P h . D$. Thesis, Punjab Agril. Univ. Ludhiana (India).

Meena, D., Tiwari, R. and Singh, O. P. (2014) Effect of nutrient spray on growth, fruit yield and quality of aonla. Annals of
Plant and Soil Research. 16 (3):242245.

Panse,V. S. and Sukhatme, P. V. (1989). Statistical Methods for Agricultural Workers, ICAR, New Delhi.

Singaram P., Prabu, P. C. (2001) Effect of zinc and boron on growth and quality of grapes cv. Muscat. Madras Agri. Journal 88 (4-6): 233-236.

Singh R. R. and Rajput, C. B. S. (1976) Effect of various concentrations of zinc on vegetative growth characters, flowering, fruiting and physico-chemical composition of fruits in mango (Mangifera indica L.) cv. Chausa. Haryana Journal Hort. Sci., 5 (1-2): 1014.

Veena P., Lavania, M. L. (1998) Effect of foliar sprays of iron, zinc and boron on growth and yield of papaya. South Indian Hortic. 46 (1-2):1-5.

Yadav, M. K., Patel, N. L., Parmar, B. R., Singh, K. and P. (2010) Effect of micronutrients on growth and crop duration of banana cv. Grand Nain. Vol42 (2): 162-164.

\section{How to cite this article:}

Vandana, G.M. Waghmare and Shivendu Pratap Singh Solanki. 2017. Influence of Chelated $\mathrm{Ca}$, Micronutrient and Method of Application on Vegetative Growth of Pomegranate (Punica granatum L.) cv. Bhagwa. Int.J.Curr.Microbiol.App.Sci. 6(11): 135-147.

doi: https://doi.org/10.20546/ijcmas.2017.611.018 